\title{
Family Functioning Predictors of Self-Concept and Self-Esteem in Children at Risk for Learning Disabilities in Oman: Exclusion of Parent and Gender Contribution
}

\author{
Mahmoud Mohamed Emam ${ }^{1} \&$ Usama Saad Abu-Serei ${ }^{1}$ \\ ${ }^{1}$ College of Education, Department of Psychology, Sultan Qaboos University, Sultanate of Oman \\ Correspondence: Mahmoud Mohamed Emam, College of Education, Department of Psychology, Sultan Qaboos \\ University, P.O. Box: 32, Al Khod 123, Muscat, Sultanate of Oman. E-mail: memam@squ.edu.om
}

Received: July 16, 2014 Accepted: August 29, 2014 Online Published: September 26, 2014

doi:10.5539/ies.v7n10p89

URL: http://dx.doi.org/10.5539/ies.v7n10p89

\begin{abstract}
The study investigated whether family functioning can predict the self-concept and self-esteem of normally achieving (NA) and at risk for learning disabilities (LD) students in Oman regardless of parent education level and gender status. A total of 259 elementary school students were selected from schools in the main districts of Muscat, the capital and largest city in Oman. The participants included 259 students referred for learning disabilities (78) and normally achieving students (181). Self-Report Measure of Family Functioning-Child Revised, Beck Self-Concept Inventory for Youth (BSCI-Y), and Rosenberg Self-Esteem Scale were administered to the participants. In addition, demographic data on parents' education levels (PEL) and gender were collected. The study specifically investigated whether family functioning dimensions of communication, cohesion, conflict, and social/recreational orientation can predict the self-concept and self-esteem of children regardless of PEL and gender status. Multiple hierarchical regressions showed that family functioning was a strong contributory factor of self-concept for both children with and without LD although the two groups differed in terms of the significant family functioning predictors. Family functioning was a weak contributory factor of self-e esteem in children with and without LD and the two groups varied in terms of the significant family functioning variables. The differences between the two groups are discussed from cultural and ecological perspectives.
\end{abstract}

Keywords: family functioning, LD, self-concept, self-esteem, Oman

\section{Introduction}

Although children with learning disabilities (LD) are often classified as a heterogeneous group, they interact dynamically within specific social contexts such as schools and families. An important aspect of the family environment is family functioning which is regarded by a number of researchers as being related to the social functioning of children with LD (Dyson, 2003). Research has shown that the majority of children with LD come from ill-structured families with disordered management styles and excessive pressures (Al-Yagon \& Mikulincer, 2004).

Given that academic difficulties expose children with LD to social and emotional difficulties, concerns have given researchers a thrust to investigate their social development (Dyson, 2003). Aspects of the social development include, among others, the development of children's self-concept and self-esteem. Research studies, however, have reached inconclusive findings regarding both areas of social development of children with LD. This is the reason that has given the researchers a thrust for further investigation of these two aspects (Dyson, 2003).

Furthermore, demographic variables such as socio-economic status and gender have been of interest in the study self-concept and self-esteem in relation to both family functioning and academic performance of children with and without LD (Gonzalez-Pienda et al., 2002). Previous research studies showed that socio economic status is associated with parents' educational level (PEL) (Mansfield, Dealy, \& Keitner, 2013). Khan, Khan and Zubairi (cited in Chohan \& Khan, 2010) argued that interacting with the child and sharing him/her in daily activities is associated with PEL. Research on the effect of gender on self-concept and self-esteem with and without LD has yielded conflicting results as did the research on the effect of PEL on family functioning (Chohan \& Khan, 2010; 
Fan, 2001; Gonzalez-Pienda et al., 2002). Using self report measures, the current study aims to explore the relationships among perceived family functioning, self-concept, and self-esteem of students at risk for LD and normally achieving (NA) children regardless of the effect of PEL and gender.

\subsection{Self-Concept}

Self-concept refers to how the individual evaluates himself/herself (Coopersmith, 1967). It provides a mediating influence on behavior and is a critical factor in the learning process (Al-Yagon, 2007). The self-concept is argued to be a set of planned self-attitudes that are relatively established and which characterize an individual (Dyson, 2003). It also refers to the individual's appraisal of worthiness of the self. Self-concept tends to reflect the social and emotional adjustment of children and adults (Harter, Whitesell, \& Junkin, 1998). A number of research studies have indicated that parental support factors contribute to the development of positive self-concept that is related to students' academic achievement (Gonzalez-Pienda et al., 2002). A number of studies have found self-concept and academic achievement are likely to predict on another (Elbaum, 2002), a higher self-concept is likely to be associated with higher academic performance (Chohan \& Khan, 2010). Research studies have come to discrepant results with regard to children with LD's self concept. A number of studies indicated that children with LD possess a low global self-concept (Gan \& Kenny, 2003) others have found no differences between children with and without LD (Gans \& Kenny, 2003). Only one study found that children with LD have a higher global self-concept than children without LD (Dyson, 2003).

Research studies which investigated gender differences in self-concept generally provided conflicting findings. In a meta-analytic study by Kling, Hyde, Showers, and Buswell (1999) the authors concluded that boys showed higher global self-concept than girls, although the difference was very small. Another study by Harter et al. (1998) showed that girls compared to boys, displayed much lower perceptions of their physical appearance. Alternatively, however, Knox, Funk, Elliot, and Bush (1998) found no significant difference between male and female high school students. Despite these findings, the literature lacks studies examining the relationship between gender and self-concept in children at risk for LD. In the current study we attend to this gap and control for gender and PEL in order to evaluate the relative importance of family functioning in relation to at risk for LD children's self-concept and self-esteem.

\subsection{Self-Esteem}

The effect of family relationships has been an important area of study in the field of social psychology. Parental relationships were argued to play a key role in determining the self-esteem of children, defined by Freshbach and Weiner (1991) as the extent to which an individual positively or negatively values his/her attributes. Cohesive families have mutual support and understanding (Lian \& Yusooff, 2009). Members of the family need to express their needs and concerns freely (Al-Yagon \& Mikulincer, 2004). When children find their parents' communication as supportive and open they develop higher self-esteem than when their parents' communication patterns are perceived as controlling and unsupportive (Heiman, Zinck, \& Heath, 2008). A family that has good parental relationships reflects that family members are willing to negotiate and solve their problems together; and that they show concern for each other with fewer conflicts occurring among them (Heiman, Zinck, \& Heath, 2008).

A child develops a general appraisal of his/her self-worth (self-esteem) at the time of school entry (Lian \& Yusooff, 2009). Self-esteem is the perceived outcome of the discrepancy between a child's goals and accomplishments. It is also affected by the degree of emotional support the child obtains from parents and peers. Self-esteem develops out of the extent to which the child experiences success and failure in his/her life. The value is attributed to the activities at which he/she succeeds or fails, and the response he/she obtains from parents about his/her performance (Lian \& Yusooff, 2009). Research indicates that parental functioning has a positive effect on self-esteem (Mandura \& Murray, 2000).

\subsection{The Current Study}

With the rise in the identification of students with LD within the educational systems in general (Elbaum, 2002) and in the Omani educational system in particular (Emam \& Kazem, 2014), and the clear documented evidence of risk factors which threaten the long-term adjustment of individuals referred for having LD (e.g., Al-Yagon \& Mikulincer, 2004), an exploration of relationships among perceived family functioning of students at risk for LD is likely to provide a better understanding of how it is related to aspects of social adjustment including their perceived self-concept and self-esteem. Recently, more research has been conducted on family functioning of children with LD, hoping that its findings may contribute to our understanding of the social, perceptual, and communication difficulties of this group of children (Dyson, 2003; Stone, Bradley, \& Kleiner, 2002). 
The general purpose of this study was to examine the impact of family functioning variables: communication, cohesion, conflict, and social/recreational orientation within the family context of students at risk for LD on their self-concept and self-esteem. On the basis of the review of the relevant literature, the specific research objectives of this study were to: (a) explore whether family functioning variables: communication, cohesion, conflict and social/recreational orientation can predict self-concept and self-esteem in students at risk for LD and NA students, (b) contribute to the literature by using a primarily Arab sample, (c) control for the effect of socio-economic status and gender which tend to yield conflicting results in order to determine the contribution of family functioning variables to perceived self-concept and self-esteem in both groups (Kling, Hyde, Showers, \& Buswell, 1999).

\section{Method}

\subsection{Participants}

Participants of the study included 78 students at risk for LD (50 female, 28 male, mean age $11.61 \pm 0.81$ years) and 181 randomly selected NA students $(83$ male, 98 female, mean age $=11.62 \pm 0.89$ years) were recruited from elementary public schools at Muscat, the capital of Oman. The schools offered regular education while providing specialized instruction to students at risk for LD only for some time during the school day. Students at risk for LD in the current study were typically enrolled in one pull-out learning support class, and were eligible for additional drop-in support as needed. They received additional support in resource rooms: they spent an average of 8 hours per week in a resource room, where they received additional support in reading and writing by a special needs LD educator. The selected participants were predominantly Arabic-speaking indigenous Omani students. Students identified as being at risk for LD by the schools were assessed special educators and met criteria set by Local Education Authorities (LEAs) based on (a) a record of poor academic achievement in reading or writing, (b) absence of any sensory problems or impairments, and (c) a record of continued poor achievement in spite of intensive academic support. However they have not been diagnosed based on an IQ-achievement discrepancy of 1.5 standard deviations or greater. In addition, they showed inadequate performance on the Omani curriculum-based tests which assessed their reading and writing skills.

\subsection{Instruments}

\subsubsection{Self-Report Measure of Family Functioning-Child, Revised (SRMFF-CR; Stark, 2002)}

The SRMFF-CR is a 40-item self-report measure of family functioning. The measure is to be completed by children on a 5-point scale ranging from 1 (Never True) to 5 (Very True). The SRMFF-CR is the revised version of the Self-Report Measure of Family Functioning (SRMFF; Bloom, 1985, cited in Stark, 2002). The original SRMFF was modified to make the language of the measure appropriate for children (Stark, 2002). The measure was later revised to improve the wording of items and remove subscales that lacked validity. The SRMFF-CR includes information on six subscales: Communication, Conflict, Social/Recreational Orientation, Cohesion, Laissez-Faire Style, and Authoritarian Style. The four subscales used in this study were Communication, Conflict, Cohesion, and Social/Recreational orientation. Cronbach's alphas for these subscales have been reported by Greenberg, Sander, and Stark (2008) as follows: Conflict $=.74$, Communication $=.87$, and Cohesion $=.73$. For the current study, a Cronbach's alpha value of $.64, .65, .69$, and .74 was obtained for the communication, cohesion, conflict and social/recreational orientation scales respectively, which indicate good internal consistency.

\subsubsection{Beck Self-Concept Inventory for Youth (BSCI-Y)}

The BSCI-Y is one of the Beck Youth Inventories (BYI). BYI contain five scales that may be used separately or in combination to assess a child's experience of depression, anxiety, anger, disruptive behavior and self-concept (Beck, Beck, \& Jolly, 2001). The scales generally measure mental health problems in children. Each of the five inventories contains 20 statements about thoughts, feelings, or behaviors associated with emotional and social impairment in children and adolescents. The inventories are intended for use with children and adolescents between the ages of 7 and 18, and are written at a second grade reading level. Items of the BSCI-Y explore self-perceptions such as competency, potency and positive self-worth. Children describe how frequently each statement is true for them, including today. For the current study a Cronbach's alpha value of .86 was obtained for the scale, indicating good internal consistency.

\subsubsection{Rosenberg Self-Esteem Scale}

Self-Esteem was assessed using the Rosenberg Self-Esteem Scale (SES) (1965). SES is a 10-item self-report measure which evaluates global feelings of self-worth and self-acceptance. The 10 items require the respondent to directly report feelings about the self. The items are rated on a four point scale: strongly agree, agree, disagree, 
and strongly disagree. These score 1,2,3, and 4, respectively, for negative items, but they score in reverse for positive items. Total scores range from 10 to 40; the higher the score is, the higher is the level of self-esteem. A number of studies reported the SES to have good reliability and validity (Schmitt \& Allik, 2005). Schmitt and Allik (2005) reported that the component structure of RSES did not vary across 53 nations. In general, cronbach's alpha was substantial (.81) across nations supporting the internal consistency of the scale across cultural contexts. For the current study a Cronbach's alpha value of .80 was obtained for the scale, indicating good internal consistency.

\subsection{Procedures}

Prior to commencement of the study, ethical approval was sought from, and granted by, Sultan Qaboos University Human Ethics Advisory Board. Ethical guidelines were strictly adhered to throughout this study. Information about the study was sent to Local Education Authorities which in turn contacted parents to take permission for the participation of their children. The instruments were combined together in one questionnaire. Demographic information consisting of gender and parents' level of education was collected. Translation procedures of the measures were based on International Test Commission Guidelines. The forward-backward translation procedure was adopted as the most appropriate strategy. The translation process used consecutive and back-translation methods to achieve the desired adaptation (Hambleton, Merenda, \& Spielberger, 2005). A third translator with a background in educational psychology performed the back-translation without an access to the original scales.

\section{Results}

Hierarchical multiple regression was performed to investigate the family functioning scales to predict self-concept and self-esteem, after controlling for demographic factors including gender and PEL. Preliminary analyses were conducted to consider the assumptions of normality, linearity, and homoscedasticity. Moreover, the correlations amongst the predictor variables, namely family functioning styles (communication, cohesion, conflict, and social/recreational orientation) included in the study were examined and these are presented in Table 1. All correlations were weak to moderate for children with $\mathrm{LD}$, ranging between $\mathrm{r}=.18, \mathrm{p}<.05$ and $\mathrm{r}$ $=.63, \mathrm{p}<.001$. Similarly correlations were weak to moderate for NA students, ranging between $\mathrm{r}=.14, \mathrm{p}<.05$ and $\mathrm{r}=.55, \mathrm{p}<.001$. This indicates that multicollinearity was unlikely to be a problem. All predictor variables were statistically correlated with self-concept and self-esteem which indicates that the data was suitable for undertaking multiple linear regression analysis. The correlations between the predictor variables and the dependent variables (self-concept and self-esteem) were all weak to moderately strong, ranging from $\mathrm{r}=.18, \mathrm{p}$ $<.05$ to $\mathrm{r}=.56, \mathrm{p}<.001$ for students at risk for LD, and from $\mathrm{r}=.13, \mathrm{p}<.05$ to $\mathrm{r}=.57, \mathrm{p}<.001$ for NA students.

To investigate whether family functioning scales can predict self-concept in students at risk for LD as well as NA students after controlling for gender and PEL we conducted a hierarchical multiple regression of two steps. In the first step of the analysis, two predictors were entered: gender and PEL. This model was statistically significant for students at risk for LD F $(2,75)=9.30 ; \mathrm{p}<.001$ and explained $19 \%$ of variance in self-concept (Table 2). After entry of family functioning scales (communication, cohesion, conflict, and social/recreational orientation) at Step 2 the total variance explained by the model as a whole was $40 \%(\mathrm{~F}(6,71)=7.99 ; \mathrm{p}<.001)$. The introduction of family functioning scales explained additional $21 \%$ variance in self-concept, after controlling for gender and PEL (R2 Change $=.21 ; \mathrm{F}(4,71)=6.07 ; \mathrm{p}<.001)$. In the final model two out of six predictor variables were statistically significant, with cohesion and social/recreational orientation recording similar Beta values $(\beta=.28, \mathrm{p}<.05)$.

For NA students the model was statistically significant $\mathrm{F}(2,178)=7.65 ; \mathrm{p}<.01$ and explained $8 \%$ of variance in self-concept (Table 2). After entry of family functioning scales (communication, cohesion, conflict, and social/recreational orientation) at Step 2 the total variance explained by the model as a whole was $39 \%(F(6,174)$ $=18.58 ; \mathrm{p}<.001)$. The introduction of family functioning scales explained additional $31 \%$ variance in self-concept, after controlling for gender and PEL $(\mathrm{R} 2$ Change $=.21 ; \mathrm{F}(4,178)=22.22 ; \mathrm{p}<.001)$. In the final model three out of six predictor variables were statistically significant, with communication recording higher Beta values $(\beta=.33, \mathrm{p}<.001)$ than the social/recreational orientation $(\beta=.21, \mathrm{p}<.001)$ and conflict $(\beta=.15, \mathrm{p}$ $<.01)$. 
Table 1. Descriptive statistics, reliability, and correlations for all variables $(\mathrm{N}=312)$

\begin{tabular}{|c|c|c|c|c|c|c|c|c|}
\hline Variables & $\mathrm{SC}$ & SE & $\mathrm{PE}$ & Gender & $\mathrm{COM}$ & $\mathrm{COH}$ & $\mathrm{CON}$ & REC \\
\hline \multicolumn{9}{|c|}{ Students at risk for $\mathrm{LD}(\mathrm{N}=\mathbf{7 8})$} \\
\hline $\mathrm{SC}$ & 1 & & & & & & & \\
\hline SE & $63 * * *$ & 1 & & & & & & \\
\hline PEL & $.26^{* * *}$ & $.26 * * *$ & 1 & & & & & \\
\hline Gender & $.43 * * *$ & $.19 *$ & $.41 * * *$ & 1 & & & & \\
\hline $\mathrm{COM}$ & $.42 * * *$ & $.19 *$ & $.18^{*}$ & $.42 * * *$ & 1 & & & \\
\hline $\mathrm{COH}$ & .50 & $.20^{*}$ & .12 & $.32 * * *$ & $.58 * * *$ & 1 & & \\
\hline CON & -.10 & .01 & $.20^{*}$ & -.06 & $-.26^{* *}$ & $-.48 * * *$ & 1 & \\
\hline REC & $.23 * * *$ & $.410 * * *$ & $.23^{* *}$ & $.53 * * *$ & $.44^{* * *}$ & $.56^{* * *}$ & -.11 & 1 \\
\hline Mean & 59.67 & 25.17 & 2.43 & 1.65 & 25.47 & 32.21 & 12.71 & 31.97 \\
\hline SD & 11.15 & 4.78 & 1.08 & .47 & 5.23 & 6.33 & 4.17 & 6.64 \\
\hline \multicolumn{9}{|c|}{ NA Students $(\mathrm{N}=181)$} \\
\hline $\mathrm{SC}$ & 1 & & & & & & & \\
\hline SE & $31 * * *$ & 1 & & & & & & \\
\hline PEL & $.17 * *$ & -.02 & 1 & & & & & \\
\hline Gender & $.20 * * *$ & .04 & -.09 & 1 & & & & \\
\hline $\mathrm{COM}$ & $.53 * * *$ & $.14^{*}$ & -.09 & $.22 * * *$ & 1 & & & \\
\hline $\mathrm{COH}$ & $.45^{* * *}$ & .02 & .06 & $.22 * * *$ & $.57 * * *$ & 1 & & \\
\hline $\mathrm{CON}$ & $-.34 * * *$ & $.22 *$ & -.05 & $-.24 * * *$ & $-.23 * * *$ & $-.49 * * *$ & 1 & \\
\hline REC & $.49 * * *$ & $.13^{* *}$ & $.16^{* *}$ & $.13^{*}$ & $.54 * * *$ & $.55^{* * *}$ & $-.32 * * *$ & 1 \\
\hline Mean & 63.24 & 25.26 & 3.80 & 1.46 & 29.01 & 35.61 & 11.46 & 34.54 \\
\hline SD & 9.60 & 4.400 & 1.24 & .50 & 5.51 & 5.29 & 3.97 & 6.09 \\
\hline
\end{tabular}

Note: $\mathrm{SC}=$ self concept; $\mathrm{SE}=$ self-esteem; $\mathrm{PEL}=$ Parents' Educational Level; $\mathrm{COM}=$ Communication; $\mathrm{COH}=$ Cohesion; $\mathrm{CON}=$ Conflict; $\mathrm{REC}=$ Recreational; ${ }^{*} p<.05,{ }^{* *} p<.01, * * * p<.001$.

To investigate whether family functioning scales can predict self-esteem in students at risk for LD and NA students after controlling for gender and PEL we conducted a hierarchical multiple regression of two steps. In the first step of the analysis, two predictors were entered: gender and PEL. This model was statistically significant for students at risk for $\operatorname{LD~F}(2,75)=3.32 ; \mathrm{p}<.05$ and explained $8 \%$ of variance in self-esteem (Table 3 ). After entry of family functioning scales (communication, cohesion, conflict, and social/recreational orientation) at Step 2 the total variance explained by the model as a whole was $12 \%(\mathrm{~F}(6,71)=3.158 ; \mathrm{p}<.05)$. The introduction of family functioning scales explained additional $4 \%$ variance in self-esteem, after controlling for gender and PEL $(\mathrm{R} 2$ Change $=.21 ; \mathrm{F}(4,71)=2.9 ; \mathrm{p}<.05)$. In the final model one out of six predictor variables were statistically significant, with social/recreational orientation recording a Beta value $(\beta=.43, \mathrm{p}<.001)$.

For NA students the model including gender and PEL was not statistically significant. After entry of family functioning scales (communication, cohesion, conflict, and social/recreational orientation) at Step 2 the model became significant and explained $11 \%$ variance of self-esteem $(F(6,174)=3.83 ; p<.001)$. The introduction of family functioning scales explained additional $11 \%$ variance in self-esteem, after controlling for gender and PEL (R2 Change $=.11 ; \mathrm{F}(4,178)=5.61 ; \mathrm{p}<.001)$. In the final model one predictor, namely conflict was significant and recorded a Beta value $(\beta=.33, \mathrm{p}<.001)$. 
Table 2. Standardized beta values for hierarchical regression analysis examining predictors of self concept in students at risk for LD and NA students

\begin{tabular}{|c|c|c|c|c|c|c|c|}
\hline \multirow[t]{2}{*}{ Variables } & \multicolumn{7}{|c|}{ Self Concept } \\
\hline & $\mathrm{R}$ & $\mathrm{R} 2$ & R2 Change & $\mathrm{B}$ & SE & $\beta$ & $\mathrm{T}$ \\
\hline \multicolumn{8}{|c|}{ Students at risk for $\mathrm{LD}(\mathrm{N}=78)$} \\
\hline Step 1 & .44 & $.19 * * *$ & & & & & \\
\hline PEL & & & & 1.05 & 1.16 & .10 & .09 \\
\hline Gender & & & & 9.15 & 2.65 & $.39 * *$ & .35 \\
\hline Stept 2 & .63 & $.40 * * *$ & $.21 * * *$ & & & & \\
\hline PEL & & & & .82 & 1.07 & .08 & .07 \\
\hline Gender & & & & 3.08 & 2.79 & .13 & .10 \\
\hline $\mathrm{COM}$ & & & & .17 & .25 & .08 & .06 \\
\hline $\mathrm{COH}$ & & & & .49 & .25 & $.28^{*}$ & .18 \\
\hline $\mathrm{CON}$ & & & & .21 & .30 & .07 & .06 \\
\hline REC & & & & .47 & .21 & $.28^{*}$ & .20 \\
\hline \multicolumn{8}{|c|}{ NA students $(\mathrm{N}=181)$} \\
\hline Step 1 & .28 & $.08 * *$ & & & & & \\
\hline PEL & & & & 1.48 & .56 & $.19 * * *$ & 2.64 \\
\hline Gender & & & & 4.31 & 1.38 & $.22 * * *$ & 3.10 \\
\hline Stept 2 & .62 & $.39 * * *$ & $.31 * * *$ & & & & \\
\hline PEL & & & & .83 & .46 & .10 & 1.77 \\
\hline Gender & & & & 1.25 & 1.19 & .06 & 1.04 \\
\hline $\mathrm{COM}$ & & & & .58 & .13 & $.33 * * *$ & 4.31 \\
\hline $\mathrm{COH}$ & & & & .08 & .15 & .04 & .53 \\
\hline $\mathrm{CON}$ & & & & -.38 & .16 & $-.15^{*}$ & -2.25 \\
\hline REC & & & & .33 & .12 & $.21 * * *$ & 2.74 \\
\hline
\end{tabular}

Note: $\mathrm{PEL}=$ Parents' Educational Level; $\mathrm{COM}=$ Communication; $\mathrm{COH}=$ Cohesion; $\mathrm{CON}=$ Conflict; $\mathrm{REC}=$ Recreational; $* p<.05, * * p<.01, * * * p .001$.

\section{Discussion}

The current study built upon the existing literature on the relationship between children's social development and family functioning variables. The first objective was to compare family functioning predictors of self-concept and self-esteem in students at risk for LD and NA students. The family functioning predictors included reported family environment variables: communication, cohesion, conflict, and social/recreational orientation, which reflect family relationships and interaction styles within the family context. We controlled for the effect of gender and socioeconomic status as reflected by PEL. With regard to self-concept, the hierarchical regression model including both gender and PEL was significant for both students at risk for LD and NA students, as was the model including the family functioning variables.

The findings showed a difference, however, in the contribution of family functioning variables to the self-concept of students at risk for LD and NA students. Group comparisons revealed that cohesion and social/recreational orientation contributed equally and uniquely to the self-concept of students at risk for LD, while communication, social/recreational orientation, and conflict contributed respectively to the self-concept of NA students. Kupersmidt and DeRosier (2004) argued that social difficulties among children and adolescents are likely to be associated with drop out of school and experience of later adjustment problems as adults. Extending this notion to students at risk for LD it is not surprising that having a sense of cohesion and the existence of social/recreational orientation could have an impact on their self-concept. As for NA students, communication 
was the strongest predictor of self-concept. An examination of item content shows that the communication scale items measure the extent to which personal problems between family members are discussed as well as the extent of children's involvement in the decision making process within the family context. It is generally not developmentally appropriate for families to include younger children and adolescents in family decision making. This may be the case in families where students are not having any problems in school. From a cultural perspective, collective cultures tend to involve children without disabilities in decision making and by contrast choose as well as decide for children with disabilities (Gaad, 2011). This may interpret the difference in the contributing variables to the self-concept in both groups in the study. The differences between contributing family functioning variables in both groups are in line with previous research on family functioning. Early investigations exploring the family environment of young people with LD showed that mothers of children with for LD were more controlling and authoritarian (Elbaum, 2002) and less encouraging and supportive than mothers of children without LD (Al-Yagon, 2007). Margalit et al. (1999) suggested that overemphasis on control and organization is likely to act as an adaptive response against the difficulties associated with the child's disability. Further research reported that families of youth with LD are characterized with high levels of rigidity, dominance, and conflict avoidance as well as decreased cooperation, expressiveness, and independence (Heiman et al., 2008).

Table 3. Standardized beta values for hierarchical regression analysis examining predictors of self-esteem in students at risk for LD and NA students

\begin{tabular}{|c|c|c|c|c|c|c|c|}
\hline \multirow[t]{2}{*}{ Variables } & \multicolumn{7}{|c|}{ Self Esteem } \\
\hline & $\mathrm{R}$ & $\mathrm{R}^{2}$ & $\mathrm{R}^{2}$ Change & $B$ & $\mathrm{SE}$ & $\beta$ & $\mathrm{T}$ \\
\hline \multicolumn{8}{|c|}{ Students at risk for $\mathrm{LD}(\mathrm{N}=78)$} \\
\hline Step 1 & .28 & $.08^{*}$ & & & & & \\
\hline PEL & & & & .99 & .53 & .22 & 1.85 \\
\hline Gender & & & & 1.03 & 1.21 & .10 & .847 \\
\hline Stept 2 & .45 & $.21 *$ & $.12 *$ & & & & \\
\hline PEL & & & & .95 & .53 & .21 & 1.80 \\
\hline Gender & & & & -1.26 & 1.37 & -.12 & -.92 \\
\hline $\mathrm{COM}$ & & & & .03 & .12 & .04 & .30 \\
\hline $\mathrm{COH}$ & & & & -.04 & .12 & -.05 & -.33 \\
\hline $\mathrm{CON}$ & & & & -.01 & .14 & -.01 & -.08 \\
\hline REC & & & & .31 & .10 & $.43 * * *$ & 3.01 \\
\hline \multicolumn{8}{|c|}{ NA students $(\mathrm{N}=181)$} \\
\hline Step 1 & .05 & .003 & & & & & \\
\hline PEL & & & & -.08 & .26 & -.02 & -.32 \\
\hline Gender & & & & .41 & .66 & .04 & .62 \\
\hline Stept 2 & .34 & $.11 * * *$ & $.11 * * *$ & & & & \\
\hline PEL & & & & -.21 & .25 & -.06 & -.82 \\
\hline Gender & & & & .63 & .66 & .07 & .96 \\
\hline $\mathrm{COM}$ & & & & .08 & .07 & .10 & 1.13 \\
\hline $\mathrm{COH}$ & & & & .01 & .08 & .01 & .16 \\
\hline $\mathrm{CON}$ & & & & .36 & .09 & $.33 * * *$ & 3.93 \\
\hline REC & & & & .12 & .06 & .17 & 1.85 \\
\hline
\end{tabular}

Note: $\mathrm{PEL}=$ Parents' Educational Level; $\mathrm{COM}=$ Communication; $\mathrm{COH}=$ Cohesion; $\mathrm{CON}=$ Conflict; $\mathrm{REC}=$ Recreational; ${ }^{*} p<.05, * * p<.01,{ }^{* * *} p<.001$. 
In as study by Baigas (2002) families of younger children with LD were reported to score significantly worse on aspects of family functioning, including roles, behavior control, communication, affective responses, and general functioning. In contrast, Dyson (1996) found that the similarities regarding the functioning of families with and without $\mathrm{LD}$ outnumber the differences.

In addition to the pattern of group differences found in self-concept, family functioning variables explained a significant percentage, albeit minimal, of the variance in self-esteem for students at risk for LD and NA students. The hierarchical regression model including both gender and PEL was modestly significant for students at risk for LD and non significant for NA students. After entry of the family functioning variables the model was significant for both students at risk for LD and NA students. For students at risk for LD the contribution of the family function variables was minimal and the significance value remained modest. Social/recreational orientation uniquely predicted the self-esteem of students at risk for LD. For NA students the contribution of family functioning variables was minimal and strongly significant, with conflict uniquely predicting the self-esteem of NA students without. These findings are notable in many ways. First, and compared to self-concept, the prediction of self-esteem by family functioning variables was weak as reflected by variance, beta, and significance values. This may be explained in terms of the characteristics of Rosenberg Self-esteem Scale which was used in the study. A number of studies have questioned the factor structure of the scale and how different it may be across different culture (Supple \& Plunkett, 2011). Some argued for the scale measures a uni-dimensional construct whereas other argued that it measures a bi-dimensional one (positive and negative) (Supple \& Plunkett, 2011). In this study we adopted the first perspective, which could have affected our findings. Second, the findings reflect that self-concept and self-esteem are two different constructs. Both refer to salient self characteristics which are perceived by individuals as being essential; and both can be assessed globally through evaluating affective aspects of self-functioning and self-worth or through specific evaluations of role performance such as performance in reading, mathematics or other aspects of activities.

For students at risk for LD it seems that Social/recreational orientation operates as a protective factor for their self-esteem. From the perspective of academic risk and resilience (Kam \& Bámaca-Colbert, 2013), protective resources include factors that may act as a cushion against risk factors when adverse experiences exist. A protective resource is likely to have little effect for low-risk groups but higher positive impacts for high-risk groups (Kam \& Bámaca-Colbert, 2013). A good example of protective resources is the supportive family context and the perfect family functioning. Parents are likely to convey to their children feelings of concern, love, security, trust, understanding, and respect through verbal and nonverbal messages, (Kam \& Bámaca-Colbert, 2013). It seems that these messages become powerful when they are delivered through social and recreational orientation in the case of students at risk for LD and through conflict in the case of NA students. An examination of the item content for the conflict scale it appears that children strive to survive any conflict to maintain high self-esteem (Kam \& Bámaca-Colbert, 2013).

The study findings are in line with previous research which provided inconclusive findings regarding the qualitative differences in family functioning of families of children with and without LD. A number of studies sought to explain the dynamics of parent-child interactions through an examination of the role of individual child characteristics and parental characteristics including coping methods and sources of support. In a study by Al-Yagon (2007) maternal coping resources were reported to moderate the effect of children's LD on levels of loneliness, feelings of hope, and secure attachment. Thus, effect of LD on children's social- emotional adjustment including their self-esteem was related to how their mothers coped as well as their dependence on social support within the family context. Further research suggested that children with learning difficulties are more susceptible to differences in parenting styles (Greenberg, Speltz, DeKlyen, \& Jones, 2001).

Parental functioning has been shown to positively influence children's self-esteem (Mandura \& Murray, 2000). In a study by Brody and Flor (1997) self-esteem was associated with family characteristics and routines as well as parent-child relationship quality. Thus children who perceive the presence of supportive family are possess good quality self-esteem and that an increase in the quality of family functioning is always paired with higher self-esteem among children (Lian, Yusoof, 2009; Mandura \& Murray, 2000). Our study findings extended previous research in terms of the aspects of family functioning that have an impact on the self-esteem of children at risk for LD and NA students. Although Mandura and Murray (2000) argued that there is a consensus across cultures regarding the magnitude of the relationship between family functioning and self-esteem, there is a need to investigate how this family functioning impact self-esteem in different cultures. In our study we highlighted and quantified the aspects that showed such impact. The same argument applied notably to the study findings on self-concept. Although we agree with Mandura and Murray that family functioning has a crucial influence on the student's self-esteem regardless of culture, yet culture may show an impact on how family functioning operates 
to have such influence.

\section{Conclusion and Implications}

There are several methodological issues which should be considered in interpreting the study findings. First, the LD sample included a variety of students at risk for LD, without sub-grouping by types (e.g., reading, writing, mathematics), which may affect self-concept. Second we used children's self-report and assumed that this would provide an accurate assessment of their self-concept, and self-esteem. Previous research, however, revealed an inconsistency between the self-assessments of LD students and their teachers' perceptions (Elbaum, 2002). Other research found that children's self-perceptions parents and teachers' perceptions were discrepant (Stone et al., 2002).

However, future research should focus on longitudinal studies and employ larger samples of Omani children who are diagnosed with LD as well as those at risk for LD from different age groups, i.e. from early childhood to early adolescence. Future research could rely on the ratings of teachers and parents in order to compare them with child, teacher, and parent perceptions. According to Kling et al. (1999) the assessment of self-concept should be through observatory rather than self-report measures.

To summarize, our findings indicate that learning disability has a significant influence on children's self-concept and self-esteem. It is imperative to determine the effects of learning disability on the child in order to adopt corrective strategies to decrease any possible negative consequences. The study findings suggest that within the home context, learning disability has various effects on children's. Although the disability is unlikely to damage children's self-concept and self-esteem, it appears to have an impact on how the family functions. Therefore, variation in self-concept and self-esteem tends to be associated with the level of family functioning and how the family is oriented to the child's disability - the social/recreational orientation was shown to determine children's self-concept and self-esteem. This finding should be considered when home-based interventions are planned for students at risk for LD.

\section{Acknowledgements}

This research was funded by the His Majesty's Research Trust Fund (HMRTF) at Sultan Qaboos University under grant No. (SR/EDU/PSYC/12/01). The authors, therefore, acknowledge with thanks HMRTF technical and financial support.

\section{References}

Al-Yagon, M. (2007). Social-emotional and behavioral adjustment among school-age children with learning disabilities: The moderating role of maternal personal resources. Journal of Special Education, 40, 205-217.

Al-Yagon, M., \& Mikulincer, M. (2004). Patterns of close relationships and social-emotional and academic adjustment among school-age children with learning disabilities. Learning Disabilities Research and Practice, 19, 12-19.

Baigas, H. R. (2002). Families and learning in classified and nonclassified first graders. Dissertation Abstracts International Section A: Humanities and Social Sciences, 63(2-A). (UMI No. 3045072)

Beck, J., Beck, A., \& Jolly, J. (2001). Manual for the Beck Depression Inventories of emotional and social adjustment. San Antonio, TX: Psychological Corporation.

Brody, G. H., \& Flor, D. L. (1997). Maternal psychological functioning, family processes, and child adjustment in rural, single-parent, African American families. Developmental Psychology, 33, 1000-1011. http://dx.doi.org/10.1037/0012-1649.33.6.1000

Chohan, B., \& Khan, R. (2010). Impact of Parental Support on the Academic Performance and Self Concept of the Student. Journal of Research and Reflections in Education, 4(1), 14-26.

Coopersmith, S. (1967). The antecedents of self-esteem. San Francisco, CA: N. H. Freeman.

Dyson, L. (1996). The Experiences of Families of Children with Learning Disabilities: Parental Stress, Family Functioning, and Sibling Self-Concept. Journal of Learning Disabilities, 29(3), 280-286.

Dyson, L. L. (2003). Children with learning disabilities within the family context: A comparison with siblings in global self-concept, academic self-perception, and social competence. Learning Disabilities Research and Practice, 18, 1-9. http://dx.doi.org/10.1111/1540-5826.00053

Elbaum, B. (2002). The self-concept of students with learning disabilities: A meta-analysis of comparisons across different placements. Learning Disabilities Research \& Practice, 17, 216-226. http://dx.doi.org/10.1111/1540-5826.00047 
Emam, A., \& Kazem, A. (2014). Visual motor integration in children with and without reading disabilities in Oman. Social and Behavioural Sciences, 112, 548-556. http://dx.doi.org/10.1016/j.sbspro.2014.01.1201

Freshbach, S., \& Weiner, L. (1991). Evolution of the African-American annals of New York. Academy of Science, $567,3-7$.

Gaad, E. (2011). Inclusive Education in the Middle East. London: Routledge.

Gans, A. M., \& Kenny, M. C. (2003). Comparing the self-concept of students with and without learning disabilities. Journal of Learning Disabilities, 36, 287-295. http://dx.doi.org/10.1177/002221940303600307

Gonzalez-Pienda, J. A, Nunez, J. C., Gonzalez-Pumariega, S., Alvarez, L., Roces, C., \& Garcia, M. (2002). A Structural Equation Model of Parental Involvement, Motivational and Aptitudinal Characteristics, and Academic Achievement. The Journal of Experimental Education, 70(3), 257-287. http://dx.doi.org/10.1080/00220970209599509

Greenberg, M. T., Speltz, L., DeKlyen, M., \& Jones, K. (2001). Correlates of clinical referral for early conduct problems: Variable and person-oriented approach. Development and Psychopathology, 13, 255-276. http://dx.doi.org/10.1017/S0954579401002048

Hambleton, R., Merenda, P., \& Spielberger, C. (2005). Adapting educational and psychological tests for cross-cultural assessment. Hillsdale, NJ: Lawrence Erlbaum.

Harter, S., Whitesell, N. R., \& Junkin, L. J. (1998). Similarities and differences in domain-specific and global self-evaluations of learning-disabled, behaviorally disordered, and normally achieving adolescents. American Education Research Journal, 35, 653-680. http://dx.doi.org/10.2307/1163462

Heiman, T., Zinck, L., \& Heath, N. (2008). Parents and youth with learning disabilities perceptions of relationships and communication. Journal of Learning Disabilities, 41(6), 524-534. http://dx.doi.org/10.1177/0022219408317860

Kam, J., \& Bámaca-Colbert, M. (2012) Supportive parenting as a moderator of perceived ethnic/racial discrimination's associations with psychological and academic Adjustment: A Comparison Between Mexican- Origin Females in Early and middle adolescence. Communication Research, 40(5), 645-668.

Kling, K. C., Hyde, J. S., Showers, C. J., \& Buswell, B. (1999). Relations of friendship quality to self-esteem in early adolescence. Journal of Early Adolescence, 16, 110-129.

Knox, M., Funk, J., Elliot, R., \& Bush, G. E. (1998). Adolescents' possible selves and their relationship to global self-esteem. Sex Roles, 31, 61-77.

Kupersmidt, J. B., \& DeRosier, M. E. (2004). How peer problems lead to negative outcomes: An integrative mediational model. In J. B. Kupersmidt, \& K. A. Dodge (Eds.), Children's peer relations (pp. 119-138). Washington, DC: American Psychological Association.

Lian, T., \& Yusoof, F. (2009). The effects of family functioning on self-esteem of children. European Journal of Social Sciences, 9(4), 643-650.

Mansfield, A., Dealy, J., \& Keitner, G. (2013). Family functioning and income: Does low-income status impact family functioning? The Family Journal, 21(3), 297-305 http://dx.doi.org/10.1177/1066480713476836

Margalit, M., Tur-Kaspa, H., \& Most, T. (1999). Reciprocal nominations, reciprocal rejections and loneliness among students with learning disorders. Educational Psychology, 19, 79-90.

Schmitt, D. P., \& Allik, J. (2005). Simultaneous administration of the Rosenberg self-esteem scale in 53 nations: Exploring the universal and culture-specific features of global self-esteem. Journal of Personality and Social Psychology, 89, 623-642. http://dx.doi.org/10.1037/0022-3514.89.4.623

Stark, K. D. (2002). The self-report measure of family functioning-child revised (Unpublished manuscript). University of Texas at Austin.

Stone, C. A., Bradley, K., \& Kleiner, J. (2002). The role of parental perceptions and interaction patterns in the creation of opportunities for children with language/learning disabilities. In B. Wong, \& M. Donahue (Eds.), The social dimensions of learning disabilities (pp. 133-160). Mahwah, NJ: Lawrence Erlbaum.

Supple, A., \& Plunkett, S. (2011). Dimensionality and validity of the Rosenberg self-esteem scale for use with Latino adolescents. Hispanic Journal of Behaviorual Sciences, 33(1), 39-53. http://dx.doi.org/10.1177/0739986310387275 


\section{Copyrights}

Copyright for this article is retained by the author(s), with first publication rights granted to the journal.

This is an open-access article distributed under the terms and conditions of the Creative Commons Attribution license (http://creativecommons.org/licenses/by/3.0/). 